\title{
Microcirculatory perfusion disturbances following cardiac surgery with cardiopulmonary bypass are associated with in vitro endothelial hyperpermeability and increased angiopoietin-2 levels
}

\author{
Nicole A. M. Dekker ${ }^{1,2,3^{*}}$ (D), Anoek L. I. van Leeuwen ${ }^{1,2,3}$, Willem W. J. van Strien ${ }^{1}$, Jisca Majolée ${ }^{2}$, Robert Szulcek ${ }^{2,4}$,
} Alexander B. A. Vonk ${ }^{3,2}$, Peter L. Hordijk2, Christa Boer ${ }^{1}$ and Charissa E. van den Brom ${ }^{1,2}$

\begin{abstract}
Background: Endothelial hyperpermeability following cardiopulmonary bypass (CPB) contributes to microcirculatory perfusion disturbances and postoperative complications after cardiac surgery. We investigated the postoperative course of renal and pulmonary endothelial barrier function and the association with microcirculatory perfusion and angiopoietin-2 levels in patients after CPB.

Methods: Clinical data, sublingual microcirculatory data, and plasma samples were collected from patients undergoing coronary artery bypass graft surgery with CPB $(n=17)$ before and at several time points up to $72 \mathrm{~h}$ after CPB. Renal and pulmonary microvascular endothelial cells were incubated with patient plasma, and in vitro endothelial barrier function was assessed using electric cell-substrate impedance sensing. Plasma levels of angiopoietin-1,-2, and soluble Tie2 were measured, and the association with in vitro endothelial barrier function and in vivo microcirculatory perfusion was determined.
\end{abstract}

Results: A plasma-induced reduction of renal and pulmonary endothelial barrier function was observed in all samples taken within the first three postoperative days $(P<0.001$ for all time points vs. pre-CPB). Angiopoietin-2 and soluble Tie2 levels increased within $72 \mathrm{~h}$ after CPB $(5.7 \pm 4.4$ vs. $1.7 \pm 0.4 \mathrm{ng} / \mathrm{ml}, P<0.0001$; $16.3 \pm 4.7$ vs. $11.9 \pm 1.9 \mathrm{ng} / \mathrm{ml}, P=0.018$, vs. pre-CPB), whereas angiopoietin-1 remained stable. Interestingly, reduced in vitro renal and pulmonary endothelial barrier moderately correlated with reduced in vivo microcirculatory perfusion after CPB $(r=0.47, P=0.005 ; r=0.79, P<0.001)$. In addition, increased angiopoietin-2 levels moderately correlated with reduced in vitro renal and pulmonary endothelial barrier $(r=-0.46, P<0.001 ; r=-0$. $40, P=0.005)$ and reduced in vivo microcirculatory perfusion $(r=-0.43, P=0.01 ; r=-0.41, P=0.03)$.

\footnotetext{
* Correspondence: n.dekker@vumc.nl

${ }^{1}$ Amsterdam UMC, Vrije Universiteit Amsterdam, Anesthesiology, Amsterdam

Cardiovascular Sciences, Amsterdam, The Netherlands

${ }^{2}$ Amsterdam UMC, Vrije Universiteit Amsterdam, Physiology, Experimental

Laboratory for Vital Signs, Amsterdam Cardiovascular Sciences, Amsterdam,

The Netherlands

Full list of author information is available at the end of the article
}

(c) The Author(s). 2019 Open Access This article is distributed under the terms of the Creative Commons Attribution 4.0 International License (http://creativecommons.org/licenses/by/4.0/), which permits unrestricted use, distribution, and reproduction in any medium, provided you give appropriate credit to the original author(s) and the source, provide a link to the Creative Commons license, and indicate if changes were made. The Creative Commons Public Domain Dedication waiver (http://creativecommons.org/publicdomain/zero/1.0/) applies to the data made available in this article, unless otherwise stated. 
(Continued from previous page)

Conclusions: CPB is associated with an impairment of in vitro endothelial barrier function that continues in the first postoperative days and correlates with reduced postoperative microcirculatory perfusion and increased circulating angiopoietin-2 levels. These results suggest that angiopoietin-2 is a biomarker for postoperative endothelial hyperpermeability, which may contribute to delayed recovery of microcirculatory perfusion after CPB.

Trial registration: NTR4212.

Keywords: Cardiopulmonary bypass, Angiopoietin-2, Capillary permeability, Microcirculation, Endothelium

\section{Background}

Cardiac surgery with cardiopulmonary bypass $(\mathrm{CPB})$ is often complicated by tissue edema as a consequence of a systemic inflammatory response and vascular endothelial hyperpermeability [1-3]. We previously showed that this impairment of endothelial barrier function and subsequent fluid shift hampers microcirculatory perfusion [4-6] and contributes to the development of postoperative organ dysfunction, in particular acute kidney and lung injury [7].

The angiopoietin/Tie2 system has been proposed as a key signaling pathway in CPB-related endothelial hyperpermeability [8-11]. Tie2 is a vascular restricted tyrosine kinase receptor with specificity for angiopoietin-1 and angiopoietin-2 binding [12]. In quiescence, angiopoietin-1 binds to Tie2, resulting in receptor phosphorylation and inhibition of inflammation. During stress as observed in $\mathrm{CPB}$, stored angiopoietin-2 is released from Weibel-Palade bodies and competes with angiopoietin-1 for Tie2 binding which antagonistically reduces endothelial barrier function and increases inflammation [12].

The potential of angiopoietin-2 as a biomarker for endothelial dysfunction and unfavorable outcome has been extensively investigated in septic populations [13-15], but has been restricted to in vitro models $[3,8]$ or the evaluation of plasma markers in cardiac surgery patients $[9,10,16]$. Increased plasma angiopoietin-2 levels following $\mathrm{CPB}$ are associated with prolonged mechanical ventilation [8] and acute kidney injury [16]. We and others showed that the onset of CPB is associated with an acute impairment of in vitro endothelial barrier function $[3,8]$. In addition, we previously showed that targeting Tie2 with an angiopoietin-1 mimetic could reduce pulmonary vascular leakage and preserve in vivo microcirculatory perfusion during and after $\mathrm{CPB}$ in an experimental model [5], implying the importance of angiopoietin1-dependent Tie2 signaling and endothelial integrity to maintain microcirculatory perfusion and organ function after $\mathrm{CPB}$.

Although recent studies emphasized the biological and clinical relevance of increased angiopoietin-2 levels in the first hours following $\mathrm{CPB}$ [8-10], the connection between postoperative angiopoietin-2 levels, endothelial barrier function, and microcirculatory perfusion following $\mathrm{CPB}$ remains to be elucidated. We therefore aimed to investigate the postoperative effects of cardiac surgery with $\mathrm{CPB}$ on in vitro renal and pulmonary endothelial barrier function and their relation with circulating angiopoietin/Tie2 and microcirculatory perfusion.

\section{Methods}

\section{Study design}

The GlyCar study was approved by the Human Subjects Committee of the Amsterdam UMC (13.291, NTR4212, Amsterdam, The Netherlands), and clinical data were previously published [6]. Patients (age 18-85 years) scheduled for elective coronary artery bypass graft (CABG) surgery with cardiopulmonary bypass $(\mathrm{CPB})$ were included. Exclusion criteria were re-operation, emergency operation, patients with type 1 diabetes mellitus, a body mass index over $35 \mathrm{~kg} / \mathrm{m}^{2}$, and patients with a history of hematologic, hepatic, or renal diseases (eGFR $<50 \mathrm{ml} / \mathrm{min}$ ).

Patients underwent standard anesthesia and cardiopulmonary bypass protocols as described previously [6] Briefly, anesthesia was induced using intravenous sufentanil $(1-3 \mu \mathrm{g} / \mathrm{kg})$, combined with rocuronium $(0.5-1.0 \mathrm{mg} / \mathrm{kg})$ and midazolam $(0.1 \mathrm{mg} / \mathrm{kg})$, and maintained by continuous propofol infusion $(100-400 \mathrm{mg} / \mathrm{h})$. The extracorporeal circuit consisted of a centrifugal blood pump and a heater cooler device (Stockert Instrumente GMBH, Munich, Germany), a phosphorylcholine-coated tubing system (P.h.i.s.i.o., The Sorin Group, Mirandola, Italy), and a hollow fiber oxygenator (Affinity, Medtronic, Minneapolis, MN, USA). CPB was initiated after heparin administration $(300 \mathrm{IU} / \mathrm{kg}$ ) when target activated clotting time (ACT) exceeded $480 \mathrm{~s}$, and supplementary doses were administered if necessary. CPB flow was maintained at 1.8 $2.6 \mathrm{l} / \mathrm{min} / \mathrm{m}^{2}$ with mild hypothermia $\left(34-36^{\circ} \mathrm{C}\right)$. At the end of surgery, anticoagulation with heparin was reversed using protamine in a 1:1 ratio to achieve normal ACT. A cell saver (Autolog, Medtronic, Minneapolis, USA) was used for autologous red blood cell transfusion [17].

\section{Collection of blood samples}

Arterial blood was collected after induction of anesthesia before onset of $\mathrm{CPB}$ (pre-CPB), after initiation of $\mathrm{CPB}$ (CPB), $1 \mathrm{~h}$ after weaning from CPB (post-CPB), and $24 \mathrm{~h}$ and $72 \mathrm{~h}$ following surgery $(+24 \mathrm{~h}$ and $+72 \mathrm{~h}$, respectively) and immediately centrifuged at $4.000 \mathrm{G}$ for $10 \mathrm{~min}$ 
at $4{ }^{\circ} \mathrm{C}$. Plasma supernatant was centrifuged for another $5 \mathrm{~min}$ at $12.000 \mathrm{~g}$ at $4{ }^{\circ} \mathrm{C}$ to obtain platelet-free plasma. Platelet-free plasma was snap frozen in liquid nitrogen and stored at $-80^{\circ} \mathrm{C}$. Plasma concentrations of angiopoietin-1 (DANG10), angiopoietin-2 (DANG20), and soluble Tie2 (DTE200, R\&D Systems, Biotechne, Minneapolis, MN, USA) were measured using commercially available enzyme-linked immunosorbent assays in accordance with the manufacturer's instructions.

\section{Cell culture}

Human primary glomerular endothelial cells isolated from human glomerular tissue were obtained from three healthy donors obtained from Cell biologics (H-6014G, Cell Biologics Company, Chicago, USA) and pooled and cultured on gelatin-coated T25 flasks in complete medium in an atmosphere of $95 \%$ air and $5 \% \mathrm{CO}_{2}$ at $37^{\circ} \mathrm{C}$ (Additional file 1: Supplemental methods). Human pulmonary microvascular endothelial cells were isolated from healthy lung tissue obtained from three donors during lobectomy (Amsterdam UMC - location VU University Medical Center, Amsterdam, The Netherlands) and cultured as described previously [18].

\section{Endothelial barrier function}

Electric Cell-substrate Impedance Sensing (ECIS, Applied BioPhysics, Troy, NY, USA) was used to measure impedance of endothelial cells [19]. Confluent glomerular endothelial cells or pulmonary microvascular endothelial cells were incubated for $1 \mathrm{~h}$ with $1 \%$ human serum albumin (HSA) in bare medium followed by the addition of $10 \%$ platelet-free plasma obtained from cardiac surgery patients at different time points before and after CPB as described above (Additional file 1: Supplemental methods). Resistance of endothelial monolayers was continuously measured at $4.000 \mathrm{~Hz}$ for $3 \mathrm{~h}$ until steady state was reached using ECIS software (v1.2.210.0 PC; Applied Bio-Physics). Measurements were performed in duplicate, and data were normalized to baseline.

\section{Immunofluorescence staining}

Immunofluorescence was used to visualize endothelial cell structures after exposure to plasma obtained from patients either before $(n=6)$ or after $(n=6)$ exposure to $\mathrm{CPB}$. Glomerular endothelial cells or pulmonary microvascular endothelial cells were exposed to plasma for $3 \mathrm{~h}$. Subsequently, endothelial cells were stained for VE-cadherin (SC-6458, Santa Cruz, Dallas, TX, USA) and actin (acti-stain Phalloidin670, Cytoskeleton, Denver, CO, USA). Nuclei were stained using DAPI (1:500; Thermo Fisher Scientific, Waltham, MA, USA) (Additional file 1: Supplemental methods).

\section{Microcirculatory perfusion}

Microcirculatory perfusion of all patients in this study, represented as percentage of perfused vessels (PPV, \%), has previously been reported [6]. Briefly, sublingual microcirculatory perfusion was measured using non-invasive side stream dark field (SDF) video microscopy (Capiscope HVCS-HR, KK Technology, Honiton, UK) to visualize flowing erythrocytes based on the absorbance spectrum of hemoglobin. Videos of around $10 \mathrm{~s}$ were obtained in three different sublingual areas per time point. Videos were analyzed off-line using automatic vascular analysis software (AVA 3.0, Microvision Medical, Amsterdam, The Netherlands) according to microvascular scoring recommendations by De Backer et al. [20]. Vessels were manually identified and scored for flow. Micro-vessels (diameter ranging from 5 to $25 \mu \mathrm{m}$ ) scored with absent or intermittent flow (at least $50 \%$ of the time absent flow) were classified as non-perfused, and micro-vessels scored with continuous flow were classified as perfused vessels. Subsequently, the proportion of perfused vessels (PPV; in \%) was automatically calculated as the proportion of perfused micro-vessels from the total amount of identified micro-vessels.

\section{Statistical analysis}

Data were analyzed with GraphPad Prism 7.0 (GraphPad Software, La Jolla, CA, USA). At least a $25 \%$ reduction $(\Delta=250 \Omega)$ in in vitro endothelial resistance was expected following exposure to post-CPB plasma with a standard deviation of $150 \Omega$ [3]. With a significance level $(\alpha)$ of 0.05 and beta of 0.9 group sizes of $n=8$ were calculated. Data are presented as mean \pm standard deviation (SD). Changes in endothelial resistance over time were evaluated using repeated measures ANOVA with Bonferroni post-hoc analyses. Two-sided paired t-test were used to evaluate differences between time points. Correlations between circulating angiopoietin-2 levels, endothelial barrier, and microcirculatory perfusion were analyzed using a Pearson correlation test. A $P$ value of $<0.05$ was considered statistically significant.

\section{Results}

\section{Patient characteristics}

A total of 17 cardiac surgery patients were included in the study. Patient characteristics are listed in Table 1. Patients had a mean age of $67 \pm 7$ years and were exposed to $\mathrm{CPB}$ for $103 \pm 18 \mathrm{~min}$ with a mean surgical time of $239 \pm 38 \mathrm{~min}$. Two patients developed de novo atrial fibrillation, and one patient developed postoperative pulmonary embolisms. No patient developed acute kidney injury, required repeat surgery, or died within 30 days after surgery. 
Table 1 Patient characteristics and intraoperative and postoperative details

\begin{tabular}{|c|c|}
\hline Characteristic & Value \\
\hline Age (years) & $67 \pm 7$ \\
\hline Male sex $(\%)$ & $15 / 17(88)$ \\
\hline Body mass index $\left(\mathrm{kg} / \mathrm{m}^{2}\right)$ & $29 \pm 4$ \\
\hline Diabetes mellitus II (\%) & $2 / 17(12)$ \\
\hline Hypertension (\%) & $5 / 17(29)$ \\
\hline Preoperative lactate (mmol/l) & $87 \pm 20$ \\
\hline Preoperative hemoglobin (mmol/l) & $8.4 \pm 0.9$ \\
\hline \multicolumn{2}{|l|}{ Intraoperative details } \\
\hline Surgery time (min) & $239 \pm 38$ \\
\hline Cardiopulmonary bypass time (min) & $103 \pm 18$ \\
\hline Aortic cross-clamp time (min) & $70 \pm 14$ \\
\hline Anastomoses $(n)$ & $3(2-4)$ \\
\hline Hemoglobin after onset of CPB (mmol/l) & $5.5 \pm 0.6$ \\
\hline Packed red blood cell transfusion (\%) 2 / 17 (12) & 12 \\
\hline Fresh frozen plasma transfusion (\%) 0 / $17(0)$ & 0 \\
\hline Thrombocytes (5-donor concentrate; \%) 3 / 17 (18) & 18 \\
\hline Cell saver transfusion (ml) & $491 \pm 119$ \\
\hline \multicolumn{2}{|l|}{ Postoperative details } \\
\hline Lactate after 24 h (mmol/l) & $1.9 \pm 0.9^{*}$ \\
\hline Hemoglobin after $72 \mathrm{~h}$ (mmol/l) & $7.0 \pm 1.0^{*}$ \\
\hline Intensive care length of stay (days) & $1(1-1)$ \\
\hline Atrial fibrillation (\%) & $2 / 17(12)$ \\
\hline Pulmonary embolisms (\%) & $1 / 17(6)$ \\
\hline
\end{tabular}

Values represent frequencies, means \pm standard deviation, or median with interquartile range

${ }^{*} P<0.05$ versus before cardiopulmonary bypass

Cardiopulmonary bypass-induced renal and pulmonary endothelial hyperpermeability persist in the first postoperative days

Plasma obtained immediately after weaning from $\mathrm{CPB}$ reduced renal endothelial barrier function by $17 \%$ compared to plasma that was obtained from these patients before CPB (Fig. 1a, b). This reduction in renal endothelial barrier function was even stronger following plasma exposure obtained at $24 \mathrm{~h}$ and $72 \mathrm{~h}$ after surgery $(0.52 \pm 0.14$ vs. $0.77 \pm 0.04, P<0.001$ and 0.52 \pm 0.11 vs. $0.77 \pm 0.04, P<0.001$; Fig. $1 \mathrm{a}, \mathrm{b})$. In pulmonary endothelial cells, a more severe reduction in endothelial barrier function of $34 \%$ was evoked following exposure to plasma obtained after weaning from $\mathrm{CPB}(0.24 \pm 0.03$ vs. $0.73 \pm 0.02, P<0.001$ vs. pre-CPB; Fig. 1c, d). This plasma-induced reduction of pulmonary endothelial barrier function was observed in all samples taken within the first three postoperative days $(P<0.001$ for all time points vs. pre-CPB; Fig. 1c, d).
Cardiopulmonary bypass induces in vitro renal and pulmonary intercellular gap formation

Plasma obtained $72 \mathrm{~h}$ after $\mathrm{CPB}$ increased actin stress fiber formation in renal $\left(4.7 \times 10^{6} \pm 1.9 \times 10^{6}\right.$ vs. $3.3 \times 10^{6} \pm$ $1.6 \times 10^{6}$ integrated fluorescence density per cell, $P=0.0016$, Additional file 1: Figure S1A, B) and pulmonary endothelial cells $\left(3.7 \times 10^{6} \pm 2.9 \times 10^{6}\right.$ vs. $2.1 \times 10^{6} \pm 1.5 \times 10^{6}$ integrated fluorescence density per cell, $P=0.03$, Additional file 1 : Figure S2 A, B) compared to plasma obtained from these patients before $\mathrm{CPB}$. In addition, exposure to plasma obtained $72 \mathrm{~h}$ after CPB reduced VE-cadherin at cell-cell contacts in renal (Additional file 1: Figure S1 A, B) and pulmonary (Additional file 1: Figure S2 A, B) endothelial cells. Loss of junctional VE-cadherin after CPB was paralleled by increased renal and pulmonary intercellular gap formation ( $5.4 \pm 3.9$ vs. $0.1 \pm 0.1$ gaps per endothelial cell, $P<0.001$, Fig. 2 a, and $7.9 \pm 2.2$ vs. $0.1 \pm 0.1$ gaps per endothelial cell, $P<0.001$, Fig. 2b, respectively).

\section{Cardiopulmonary bypass is associated with prolonged postoperative increased angiopoietin-2 levels}

$\mathrm{CPB}$ was associated with increased circulating levels of angiopoietin-2 within $24 \mathrm{~h}$ after surgery $(4.0 \pm 1.4$ vs. $1.7 \pm 0.4 \mathrm{ng} / \mathrm{ml}, P<0.0001$ vs. pre-CPB). Circulating angiopoietin-2 levels further increased in the following 72 postoperative hours $(5.7 \pm 4.4$ vs. $1.7 \pm 0.4 \mathrm{ng} / \mathrm{ml}, P<$ 0.0001 vs. pre-CPB; Fig. 3a). In contrast, circulating angiopoietin-1 levels remained stable in the first $72 \mathrm{~h}$ after surgery $(2.6 \pm 1.2$ vs. $1.9 \pm 1.7 \mathrm{ng} / \mathrm{ml}, P>0.9$ vs. pre-CPB; Fig. 3b). A twofold rise in angiopoietin- $2 / 1$ ratio was found $72 \mathrm{~h}$ after surgery compared to pre-CPB $(2.8 \pm 2.5$ vs. $1.2 \pm 0.4, P=0.48$; Fig. 3c). Circulating levels of the soluble form of the endothelial Tie2 receptor increased $72 \mathrm{~h}$ after surgery compared to pre-CPB (16.3 \pm 4.7 vs. $11.9 \pm 1.9 \mathrm{ng} / \mathrm{ml}, P=0.018$; Fig. 3d).

Reduced in vitro renal and pulmonary endothelial barrier function are associated with increased angiopoietin-2 levels

Associations were found between plasma-induced reduction in endothelial barrier function and increased angiopoietin-2 levels at corresponding time points in patients undergoing CPB (Fig. 4a, b). During the entire study period, increased circulating levels of angiopoietin-2 correlated with plasma-induced reduction in renal and pulmonary endothelial barrier function $(r=-0.46, P=0.0006$, Fig. 4a; and $r=-0.40, P=0.005$, Fig. $4 \mathrm{~b}$, respectively).

\section{Increased angiopoietin-2 levels are associated with} postoperative microcirculatory perfusion disturbances Increased postoperative circulating angiopoietin-2 levels in patients after $\mathrm{CPB}$ correlated to reduced microcirculatory perfusion in these patients, as represented by the proportion of perfused vessels $(r=-0.43, P=0.01$, Fig. $4 \mathrm{c})$. In 


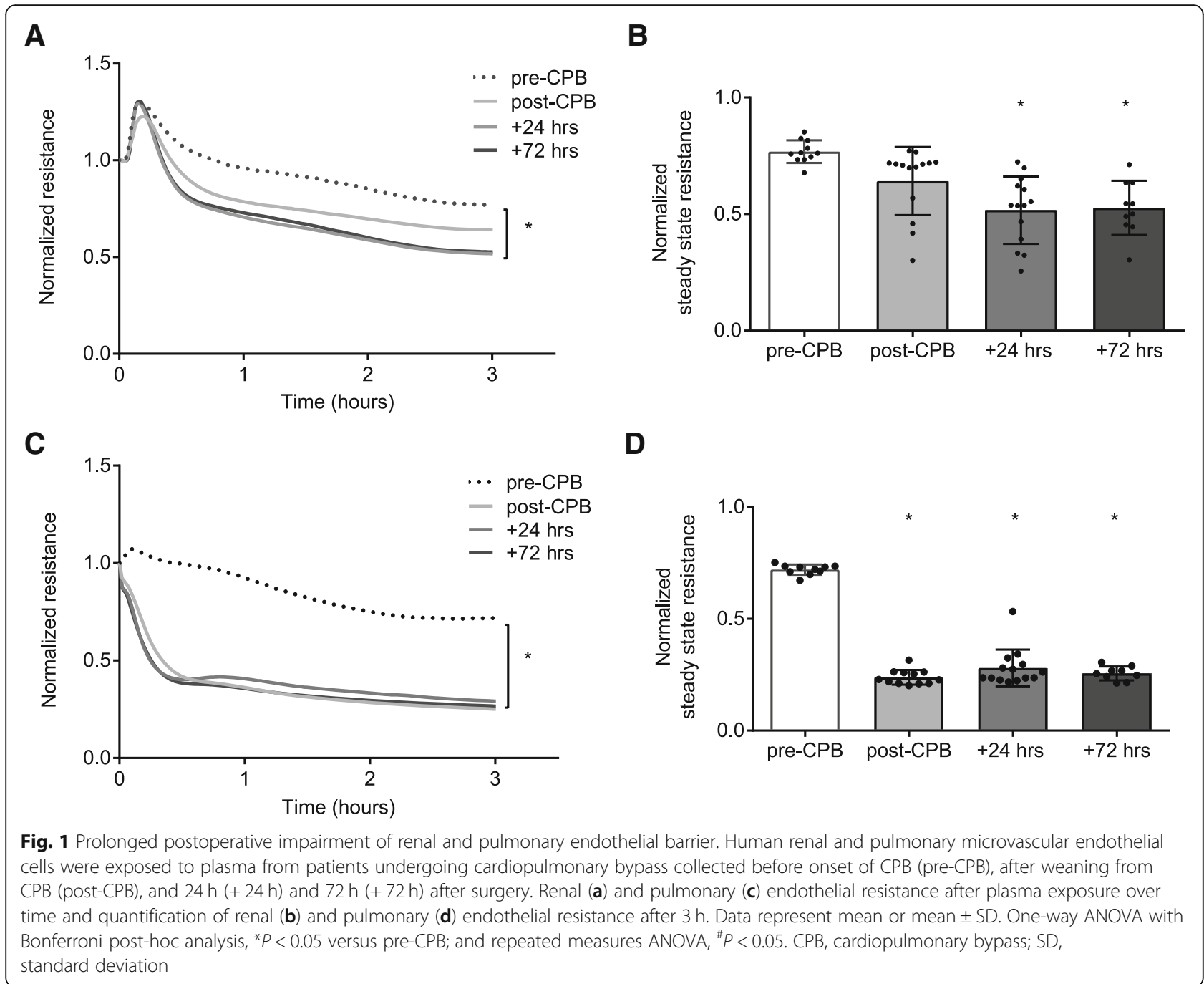

parallel, plasma lactate, a surrogate marker for impaired tissue perfusion, was positively associated with increased angiopoietin-2 levels $(r=0.63, P<0.0001$; Fig. $4 d)$. Moreover, plasma-induced reduction in renal and pulmonary endothelial barrier function was associated with reduced microcirculatory perfusion at corresponding time points in these patients $(r=0.47, P=0.005$, and $r=0.79$, $P<0.001$, respectively; Fig. 4e, f).

\section{Discussion}

In this study, we show that cardiac surgery with cardiopulmonary bypass $(\mathrm{CPB})$ is associated with a cell-type specific in vitro endothelial hyperpermeability induced by patient plasma. This plasma-induced renal and pulmonary endothelial hyperpermeability persists until at least $72 \mathrm{~h}$ after surgery and were associated with increased circulating angiopoietin-2 levels. In addition, endothelial hyperpermeability as well as increased circulating angiopoietin-2 levels following CPB correlated with in vivo microcirculatory perfusion disturbances.
These results suggest that postoperative endothelial hyperpermeability may contribute to delayed recovery of CPB-induced microcirculatory perfusion disturbances, possibly sustained by postoperative release of angiopoietin- 2 .

Endothelial hyperpermeability is increasingly recognized as a key pathophysiological contributor to postoperative organ dysfunction following cardiac surgery with CPB [7-10]. However, this parameter is clinically limited to the evaluation of fluid overload reflected in pulmonary and renal performance. Despite the growing number of clinical studies evaluating the course of circulating endothelial injury markers, evidence for a causal relation between endothelial barrier dysfunction and impaired oxygenation is scarce and mainly restricted to experimental models $[4,5]$. In line with previous studies, we found that the effects of patient plasma withdrawn following $\mathrm{CPB}$ associated with reduced in vitro endothelial barrier function. In addition, our results extend previous findings by revealing that this induced loss of endothelial barrier function can be observed in both renal and 
A

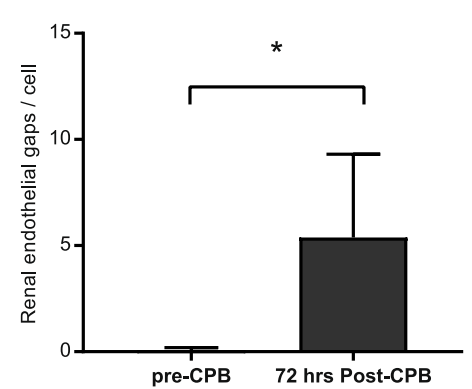

B

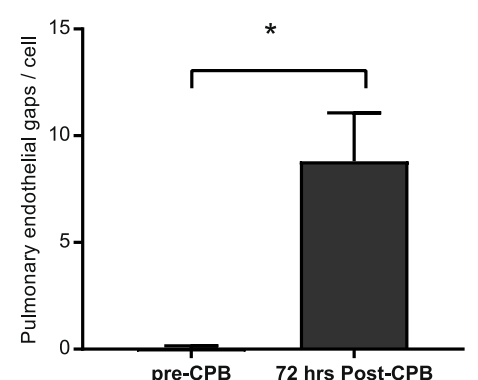

Pre-CPB

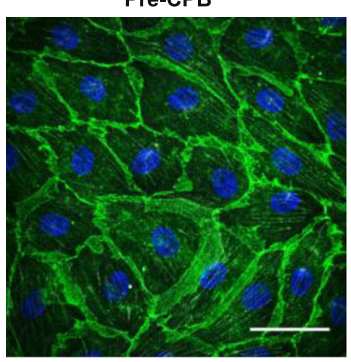

Pre-CPB

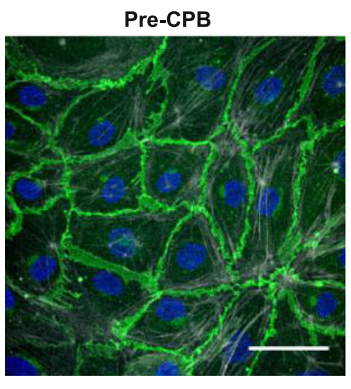

72 hrs Post-CPB

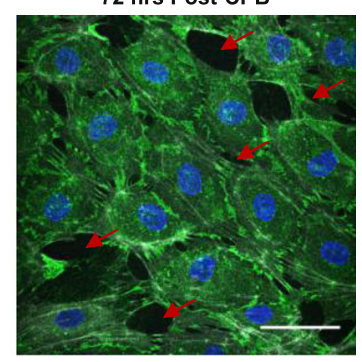

72 hrs Post-CPB

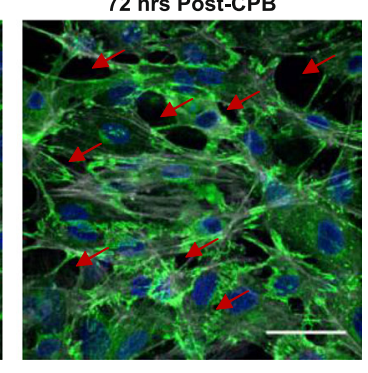

Fig. 2 Post-CPB plasma induces renal and pulmonary endothelial gap formation. Quantification of renal (a) and pulmonary (b) intercellular gap formation and representative images of endothelial cells after exposure of plasma from patients before CPB (pre-CPB, middle panels) and $72 \mathrm{~h}$ after CPB (72 h post-CPB, right panels). Endothelial cells were stained for VE-cadherin (adherens junctions; green), actin (stress fibers; white), and DAPI (nuclei; blue) after $3 \mathrm{~h}$ of plasma exposure. Red arrows indicate examples of endothelial gaps. Scale bar represents $50 \mu \mathrm{m}$. Data represent mean number of gaps per endothelial cell \pm SD quantified from $n=5$ images per time point from 6 patients. One-way ANOVA with Bonferroni post-hoc analysis, ${ }^{*} P<0.05$ versus pre-CPB. CPB, cardiopulmonary bypass; SD, standard deviation

pulmonary endothelial cells and persists in the first three postoperative days.

Vascular endothelial permeability following CPBassociated systemic inflammation is regulated by several mechanisms of which the angiopoietin/Tie2 system is postulated as central regulator [7-9]. Tie2 is an endothelium-specific transmembrane tyrosine kinase receptor, with angiopoietin-1 and angiopoietin- 2 as most dominant ligands [12]. The paracrine agonist angiopoietin-1 protects endothelial integrity by strengthening intercellular junctions. In contrast, the competitive antagonist angiopoietin-2 is released from Weibel-Palade bodies during inflammation and increases endothelial permeability. The observed endothelial barrier disruptive effect of plasma obtained after $\mathrm{CPB}$ was more severe in pulmonary endothelium compared to renal endothelium. This could be due to differences in endothelial Tie2-receptor expression levels, since Tie2 is most abundantly expressed in pulmonary microvasculature [21]. Inhibition of Tie2 via angiopoietin-2 following CPB triggers endothelial hyperpermeability by reducing junctional VE-cadherin [22], the essential component of cell-cell junctions. We indeed found that functional loss of endothelial barrier was paralleled with profound changes in cell structures, such as reduced VE-cadherin at cell-cell junctions, increased stress fiber formation, and intercellular gap formation. As both renal and pulmonary function highly depend on intact microvascular barrier, it may not be surprising that complications after $\mathrm{CPB}$ mainly present themselves in these organs $[12,13,16,23]$.

Besides its regulatory role in endothelial barrier function, angiopoietin-2 has emerged as a potential early prognostic biomarker [14, 15]. Increased circulating angiopoietin-2 has strongly been linked to the duration of mechanical ventilation, ICU length of stay, positive fluid balance, and increased postoperative organ dysfunction following $\mathrm{CPB}$ $[10,16,23]$. In this study, we observed that increased circulating angiopoietin-2 correlated not only with the endothelial barrier disruptive effect of plasma after CPB but also with in vivo microcirculatory perfusion disturbances and lactate levels. Interestingly however, the timing and trend of alterations in angiopoietin-2 do not mirror alterations in microcirculatory perfusion and lactate levels. The delayed increase in angiopoietin-2 following CPB implies that angiopoietin-mediated endothelial barrier dysfunction happens secondary to early CPB-associated endothelial dysfunction and microvascular alterations. These results may suggest that angiopoietin- 2 could be involved in prolonging the postoperative leakiness of the endothelium and thereby may attenuate restoration of microcirculatory perfusion 


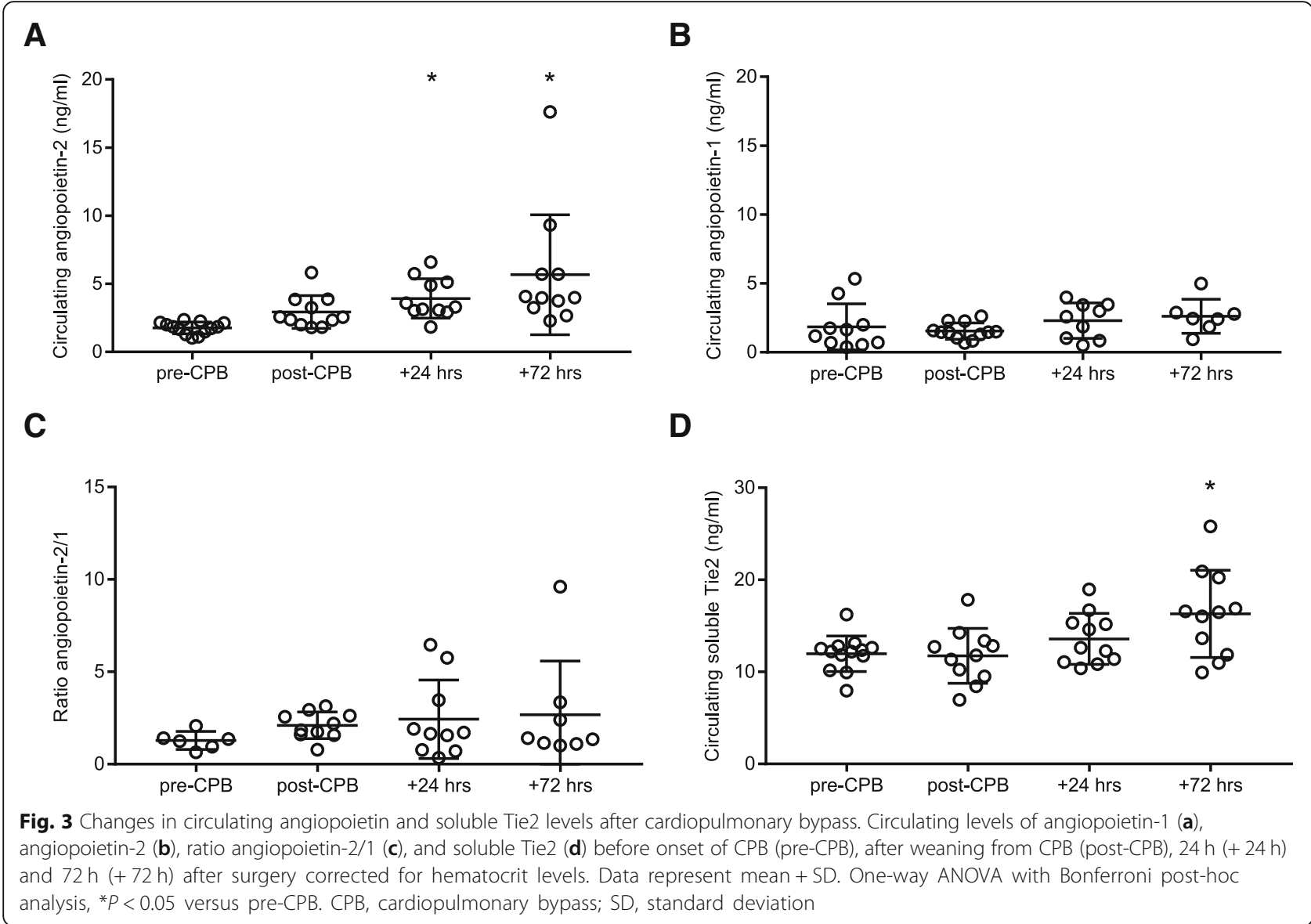

following $\mathrm{CPB}$ rather than acting as a central mediator during the onset of $\mathrm{CPB}$. In view of these divergent trends, it should also be considered that the found association between angiopoietin-2, endothelial hyperpermeability, and microcirculatory perfusion do not fit with a causal mechanism and may simply reflect a common problem, namely CPB-associated endothelial injury.

Ideally, one would like to further investigate this role of angiopoietin-2 in endothelial permeability and microcirculatory perfusion disturbances by targeting plasma angiopoietin-2 with an antibody blocking its effect leading to attenuation of our described plasma-induced hyperpermeability. Previously, this effect of angiopoietin-2 on endothelial hyperpermeability has been studied in the context of sepsis. Increased endothelial paracellular gap formation induced by serum obtained from sepsis patients could be fully neutralized by blocking angiopoietin-2 [24]. In addition, administration of an angiopoietin-2 inhibitor was found to protect endothelial integrity, reduce pulmonary vascular leakage, and improve survival in experimental sepsis models $[25,26]$. Unfortunately, these types of angiopoietin-2 inhibitors are no longer available for experimental testing, and therefore, our results should be interpreted with caution.
Remarkably, all patients in this study showed postoperative increases in angiopoietin-2 levels and plasma-induced in vitro endothelial permeability following CPB. Therefore, the next step would be to identify whether increased angiopoietin-2 may aid in identifying the patients at risk for developing complications and who may possibly benefit from therapy targeted at attenuating postoperative endothelial permeability. When interpreting our data, it is important to mention that we investigated a relatively low-risk cardiac surgery population who were rapidly discharged from the ICU. Studies investigating these alterations in high-risk cardiac surgery populations, who may possibly experience more pronounced alterations in angiopoietin- 2 and microcirculatory perfusion, would be of interest to further clarify the clinical implication of our findings.

Besides angiopoietin-2, additional barrier disruptive mediators are involved in CPB-associated endothelial hyperpermeability. Like angiopoietin-2, von Willebrand Factor is stored in Weibel-Palade Bodies and immediately released upon onset of CPB. Release of von Willebrand Factor and generation of thrombin activates coagulation, increases endothelial permeability, and stimulates release of angiopoietin-2. Besides Tie2 inhibition, activation of 


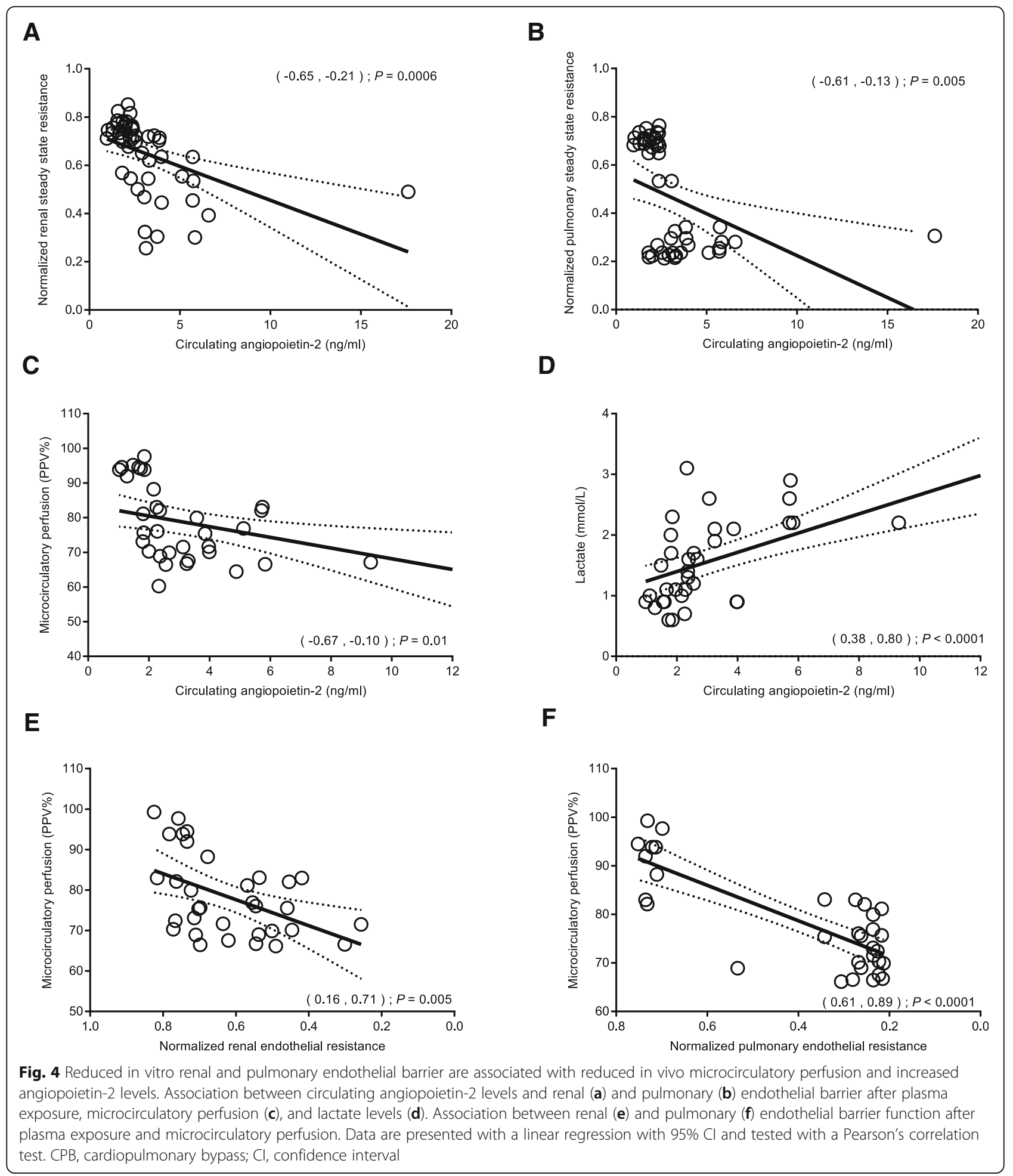

vascular endothelial growth factor receptor-2 (VEGFR2) is known to increase permeability by internalizing junctional VE-cadherins [27]. Moreover, VEGF is thought to increase permeability by promoting proteolytic cleavage and shedding of the Tie2 receptor [28]. We indeed found increased soluble Tie 2 levels at the third postoperative day, suggestive of Tie2 receptor cleavage and shedding after CPB. Altogether, multiple regulatory systems are involved in CPB-associated endothelial hyperpermeability, but all potentiate angiopoietin-2 release. Inhibition of circulating angiopoietin- 2 or stimulation of Tie 2 activity may therefore provide interesting future therapeutic targets to 
attenuate postoperative evolution of CPB-associated endothelial hyperpermeability [29-31].

\section{Conclusions}

We showed that cardiac surgery with cardiopulmonary bypass $(\mathrm{CPB})$ associated with a cell-type specific in vitro endothelial hyperpermeability induced by patient plasma. This plasma-induced renal and pulmonary endothelial hyperpermeability persisted until at least $72 \mathrm{~h}$ after surgery and corresponded to increased circulating angiopoietin-2 levels. These effects were associated with in vivo microcirculatory perfusion disturbances in corresponding patients. These results suggest that angiopoietin-2 is a biomarker for endothelial hyperpermeability which may contribute to delayed recovery of postoperative microcirculatory perfusion disturbances and the development of organ dysfunction following cardiac surgery with CPB. Whether alterations in angiopoietin-2 may help to identify patients at risk of developing complications, and who may possibly benefit from additional therapy, remains to be investigated in future studies.

\section{Additional file}

Additional file 1: Supplemental methods. Figure S1. Changes in renal endothelial cell structures following post-CPB plasma exposure. Figure S2. Changes in pulmonary endothelial cell structures following post-CPB plasma exposure. (ZIP $280 \mathrm{~kb})$

\section{Abbreviations \\ ACT: Activated clotting time; ANOVA: Analysis of variance; bM199: Bare M199 medium; CABG: Coronary artery bypass grafting; Cl: Confidence interval; CM199: Complete M199 medium; $\mathrm{CO}_{2}$ : Carbon dioxide; \\ CPB: Cardiopulmonary bypass; DAPI: 4',6-Diamidino-2-phenylindole; ECIS: Electric cell-substrate impedance sensing; EGF: Endothelial growth factor; eGFR: Estimated glomerular filtration rate; HSA: Human serum albumin; ICU: Intensive care unit; IQR: Interquartile range; PMVEC: Pulmonary microvascular endothelial cell; PPV: Proportion of perfused microvessels; SD: Standard deviation; Tie2: Tyrosine kinase with immunoglobulin-like and EGF-like domains 2; VE-cadherin: Vascular endothelial cadherin; VEGF: Vascular endothelial growth factor; VEGFR2: Vascular endothelial growth factor receptor-2}

\section{Acknowledgements}

Not applicable.

\section{Funding}

N.A.M.D. is financially supported by the Dutch Heart Foundation (Grant number 2016 T064). R.S. acknowledges financial support from the Netherlands CardioVascular Research Initiative Grant awarded to the Phaedra consortium (Grant Number 2012-08). C.E.v.d.B. is financially supported by the European Society of Anaesthesiology (Research Project Grant 2016), European Society of Intensive Care Medicine (Levi-Montalcini Award 2017), and Dutch Society of Anesthesiology (Young Investigator Grant 2017). The remaining authors are financially supported by their department. No financial support was provided from industrial companies.

\section{Availability of data and materials}

The datasets used and/ or analyzed during the current study are available from the corresponding author on reasonable request.

\section{Authors' contributions}

$\mathrm{NAMD}, \mathrm{CEv} d \mathrm{~B}, \mathrm{CB}$, and PLH were responsible for the conception and design of the study. NAMD, ALIVL, JM, WWJVS, and RS were responsible for the acquisition and analysis of the data. NAMD, ALIVL, JM, WWJvS, ABAV, CEvdB, $\mathrm{PH}, \mathrm{CB}$, and $\mathrm{RS}$ were responsible for the interpretation of the data. NAMD and CEvdB drafted the manuscript. All authors agree to be accountable for all aspects of the work in ensuring that questions related to the accuracy or integrity of any part of the work are appropriately investigated and resolved. All authors read and approved the final manuscript.

\section{Ethics approval and consent to participate}

The Glycar study was approved in the Netherlands by the Human Subjects Committee of the Amsterdam University Medical Centers under committee's reference number 13.291 (Clinical trial registration: NTR4212). Written informed consent was obtained from all patients before inclusion.

Consent for publication

Not applicable.

\section{Competing interests}

The authors declare that they have no competing interests.

\section{Publisher's Note}

Springer Nature remains neutral with regard to jurisdictional claims in published maps and institutional affiliations.

\section{Author details}

${ }^{1}$ Amsterdam UMC, Vrije Universiteit Amsterdam, Anesthesiology, Amsterdam Cardiovascular Sciences, Amsterdam, The Netherlands. ${ }^{2}$ Amsterdam UMC, Vrije Universiteit Amsterdam, Physiology, Experimental Laboratory for Vital Signs, Amsterdam Cardiovascular Sciences, Amsterdam, The Netherlands. ${ }^{3}$ Amsterdam UMC, Vrije Universiteit Amsterdam, Cardiothoracic Surgery, Amsterdam Cardiovascular Sciences, Amsterdam, The Netherlands.

${ }^{4}$ Amsterdam UMC, Vrije Universiteit Amsterdam, Pulmonology, Amsterdam Cardiovascular Sciences, Amsterdam, The Netherlands.

Received: 19 November 2018 Accepted: 1 April 2019

Published online: 11 April 2019

\section{References}

1. Hirleman E, Larson DF. Cardiopulmonary bypass and edema: physiology and pathophysiology. Perfusion. 2009;23:311-22.

2. Jongman RM, Zijlstra JG, Kok WF, van Harten AE, Mariani MA, Moser J, et al. Off-pump CABG surgery reduces systemic inflammation compared with onpump surgery but does not change systemic endothelial responses: a prospective randomized study. Shock. 2014;42(2):121-8.

3. Koning NJ, Overmars MA, van den Brom CE, van Bezu J, Simon LE, Vonk $A B A$, et al. Endothelial hyperpermeability after cardiac surgery with cardiopulmonary bypass as assessed using an in vitro bioassay for endothelial barrier function. Br J Anaesth. 2016;116(2):223-32.

4. Koning NJ, de Lange F, van Meurs M, Jongman RM, Ahmed Y, Schwarte LA, et al. Reduction of vascular leakage by imatinib is associated with preserved microcirculatory perfusion and reduced renal injury in a rat model of cardiopulmonary bypass. Br J Anaesth. 2018;120(6):1165-75.

5. Dekker NAM, van Meurs M, van Leeuwen ALI, Hofland HM, van Slyke P, Vonk ABA, et al. Vasculotide, an angiopoietin-1 mimetic, reduces pulmonary vascular leakage and preserves microcirculatory perfusion during cardiopulmonary bypass in rats. Br J Anaesth. 2018. https://doi. org/10.1016/j.bja.2018.05.049.

6. Dekker NAM, Veerhoek D, Koning NJ, van Leeuwen ALI, Elbers PWG, van den Brom CE, et al. Postoperative course of microcirculatory perfusion and endothelial glycocalyx shedding following cardiac surgery with cardiopulmonary bypass. Anaesthesia. 2018. https://doi.org/10.1111/anae.14577.

7. Brudney CS, Gosling P, Manji M. Pulmonary and renal function following cardiopulmonary bypass is associated with systemic capillary leak. J Cardiothorac Vasc Anaesth. 2005;19(2):188-92.

8. Hilbert T, Duerr GD, Hamiko M, Frede S, Rogers L, Baumgarten G, et al Endothelial permeability following coronary artery bypass grafting: an observational study on the possible role of angiopoietin imbalance. Crit Care. 2016;20:51. 
9. Giuliano JS Jr, Lahni PM, Bigham MT, Manning PB, Nelson DP, Wong $H R$, et al. Plasma angiopoietin-2 levels increase in children following cardiopulmonary bypass. Intensive Care Med. 2008;34:1851-7.

10. Clajus C, Lukasz A, David S, Hertel B, Lichtinghagen R, Parikh SM, et al. Angiopoietin-2 is a potential mediator of endothelial barrier dysfunction following cardiopulmonary bypass. Cytokine. 2012;60:352-9.

11. Benest AV, Kruse K, Savant S, Thomas M, Laib AM, Loos EK, et al. Angiopoietin2 is critical for cytokine-induced vascular leakage. PLoS One. 2013;8(8):e70459.

12. Milam KE, Parikh SM. The angiopoietin-Tie2 signaling axis in the vascular leakage of systemic inflammation. Tissue Barriers. 2015;3(1-2):e957508.

13. Parikh SM, Mammoto T, Schultz A, Yuan HT, Christiani D, Karumanchi SA, et al. Excess circulating angiopoietin-2 may contribute to pulmonary vascular leak in sepsis in humans. PLoS Med. 2006;3:e46.

14. Fisher J, Douglas JJ, Linder A, Boyd JH, Walley KR, Russell JA. Elevated plasma angiopoietin-2 levels are associated with fluid overload, organ dysfunction, and mortality in human septic shock. Crit Care Med. 2016;44(11):2018-27.

15. Ricciuto DR, dos Santos CC, Hawkes M, Toltt L, Conroy AL, Rajwans N, et al. Angiopoietin-1 and angiopoietin-2 as clinically informative prognostig biomarkers of morbidity and mortality in severe sepsis. Crit Care Med. 2011;39(4):702-10.

16. Jongman RM, van Klarenbosch J, Molema G, Zijlstra JG, de Vries AJ, van Meurs M. Angiopoietin/Tie2 dysbalance is associated with acute kidney injury after cardiac surgery assisted by cardiopulmonary bypass. PLoS One. 2015;10(8):e0136205.

17. Vonk AB, Meesters Ml, Garnier RP, Romijn JW, van Barneveld LJ, Heymans $M W$, et al. Intraoperative cell salvage is associated with reduced postoperative blood loss and transfusion requirements in cardiac surgery: a cohort study. Transfusion. 2013;53:2782-9.

18. Szulcek R, Happé CM, Rol N, Fontijn RD, Dickhoff C, Hartemink KJ, et al. Delayed microvascular shear adaptation in pulmonary arterial hypertension. Role of platelet endothelial cell adhesion molecule-1 cleavage. Am J Respir Crit Care Med. 2016;193(12):1410-20.

19. Szulcek R, Bogaard HJ, van Nieuw Amerongen GP. Electric cell-substrate impedance sensing for the quantification of endothelial proliferation, barrier function, and motility. J Vis Exp. 2014;28:85.

20. De Backer D, Hollenberg S, Boerma C, Goedhart P, Büchele G, OspinaTascon $G$, et al. How to evaluate the microcirculation: report of a round table conference. Crit Care. 2007;11(5):R101.

21. Sugiyama MG, Armstrong SM, Wang C, Hwang D, Leong-Poi H, Advani A, et al. The Tie2-agonist Vasculotide rescues mice from influenza virus infection. Sci Rep. 2015;5:11030.

22. Zhang J, Jiang Z, Bao C, Mei J, Zhu J. Cardiopulmonary bypass increases pulmonary microvascular permeability through the Src kinase pathway: involvement of caveolin-1 and vascular endothelial cadherin. Mol Med Rep. 2016;13(3):2918-24.

23. Ushida T, Ito H, Yamamoto H, Ohno N, Asahara M, Yamada Y, et al. Elevated levels of angiopoietin-2 as a biomarker for respiratory failure after cardiac surgery. J Cardiothorac Vasc Anesth. 2014;28(5):1293-301.

24. David S, Mukherjee A, Ghosh CC, Yano M, Khankin EV, Wenger JB, et al. Angiopoietin-2 may contribute to multiple organ dysfunction and death in sepsis. Crit Care Med. 2012:40(11):3034-41.

25. Han S, Lee S, Kim KE, Lee HS, Oh N, Park I, et al. Amelioration of sepsis by Tie2 activation-induced vascular protection. Sci Transl Med. 2016;8(335):335ra55.

26. Ziegler T, Horstkotte J, Schwab C, Pfetsch V, Weinmann K, Dietzel S, et al. Angiopoietin 2 mediates microvascular and hemodynamic alterations in sepsis. J Clin Invest. 2013. https://doi.org/10.1172/JCl66549.

27. Dejana E, Orsenigo F, Lampugnani MG. The role of adherence junctions and VE-cadherin in the control of vascular permeability. J Cell Science. 2008;121: 2115-22.

28. van der Heijden $M$, van Nieuw Amerongen GP, van Hinsbergh WW, Groeneveld AB. The interaction of soluble Tie2 with angiopoietins and pulmonary vascular permeability in septic and nonseptic critically ill patients. Shock. 2010;33(3):263-8.

29. Fachinger $G$, Deutsch U, Risau W. Functional interaction of vascular endothelial-protein tyrosine phosphatase with the angiopoietin recepto Tie-2. Oncogene. 2009;18:5948-53.

30. van Meurs M, Kumpers P, Ligtenberg JJ, Meertens JH, Molema G, Zijlstra JG. Bench-to-bedside review: angiopoietin signalling in critical illness - a future target? Crit Care. 2009;13(2):207.

31. Parikh SM. The angiopoietin-Tie2 signaling axis in systemic inflammation. J Am Soc Nephrol. 2017:28:197319-82.

\section{Ready to submit your research? Choose BMC and benefit from:}

- fast, convenient online submission

- thorough peer review by experienced researchers in your field

- rapid publication on acceptance

- support for research data, including large and complex data types

- gold Open Access which fosters wider collaboration and increased citations

- maximum visibility for your research: over $100 \mathrm{M}$ website views per year

At BMC, research is always in progress.

Learn more biomedcentral.com/submissions 